\title{
Assessment of Profit of a Two-Stage Deteriorating Linear Consecutive 2-out-of-3 Repairable System
}

\author{
Ibrahim Yusuf $^{*}$, Fatima Salman Koki ${ }^{2}$ \\ ${ }^{1}$ Department of Mathematical Sciences, Bayero University, Kano, Nigeria \\ ${ }^{2}$ Department of Physics, Bayero University, Kano, Nigeria \\ Email: *Ibrahimyusuffagge@gmail.com, FatimaSK2775@gmail.com
}

Received June 5, 2013; revised July 6, 2013; accepted August 15, 2013

Copyright (C) 2013 Ibrahim Yusuf, Fatima Salman Koki. This is an open access article distributed under the Creative Commons Attribution License, which permits unrestricted use, distribution, and reproduction in any medium, provided the original work is properly cited.

\begin{abstract}
Most of the researches on profit and cost evaluation of redundant system focus on the effect of failure and repair on revenue generated. However, as these systems continue to work, their strength gradually deteriorates. Where such deterioration occurs, minor and major maintenance is employed to remedy the deterioration. Little or no attention is paid on the effect of deterioration on the impact of deterioration and their maintenance on the revenue generated. In this paper, we study the profit generated of two-stage deteriorating linear consecutive 2-out-of-3 system. Failure, repair and deterioration time are assumed exponential. The explicit expressions of availability, busy period of a repairman and profit function are derived using Kolmogorov's forward equations method. Various cases are analyzed graphically to investigate the effect of deterioration parameters such as slow deterioration, fast deterioration, and their maintenance such as minor and major minimal maintenance on profit generated.
\end{abstract}

Keywords: Reliability; Availability; Profit; Deterioration

\section{Introduction}

During operation, the strengths of systems are gradually deteriorated, until some point of deterioration failure, or other types of failures. Minor and major maintenance policies are vital in the analysis of deterioration and deteriorating systems as they help in improving reliability, availability and the overall revenue generated. Both minor and major minimal maintenance are employed to check the effect of slow and fast deterioration and return the system to its state prior to slow and fast deterioration. Maintenance models assume perfect repair (as good as new), minimal repair (as bad as old) and imperfect repair which is between perfect and minimal repair. Many research results have been reported on the reliability of 2-out-of-3 redundant systems. For example, [1], analyzed reliability models for 2-out-of-3 redundant system are subject to conditional arrival time of the server. Reference [2] presented reliability and economic analysis of 2-out-of-3 redundant system with priority to repair and [3] studied MTSF and cost effectiveness of 2-out-of-3 cold standby system with probability of repair and inspection,

${ }^{*}$ Corresponding author. while [4] examined the cost benefit analysis of series systems with cold standby components and repairable service station. Reference [5,6] examined the cost analysis of two unit cold standby system involving preventive maintenance respectively. Reference [7] studied the cost and probabilistic analysis of series system with mixed standby components while [8] studied cost benefit analysis of series systems with warm standby components involving general repair time where the server is not subject to breakdowns. The failure time and repair time are assumed to have exponential distribution. Measures of system effectiveness such as MTSF, steady-state availability, busy period and profit function are obtained. [9] studied availability of a system with different repair options, while [10] evaluated the reliability of network flows with stochastic capacity and cost constraint. The problem considered in this paper is different from the work of [4-6]. In this paper, a linear consecutive 2-outof-3 repairable system with two consecutive deterioration stages (slow and fast) is studied with minor and major minimal maintenance at slow and fast deterioration respectively. In this paper, a two-stage deteriorating linear consecutive 2-out-of-3 system was constructed and derived its corresponding mathematical models. The main 
contribution of this paper is two fold. The first is to develop the explicit expressions for system availability, busy period and profit function. The second is to perform a parametric investigation of various system parameters on profit function and capture their effect on the profit function.

The rest of the paper is organized as follows. Section 2 is the description and states of the system. Section 3 deals with models formulation. The results of our numerical simulations are presented and discussed in Section 4. The paper is concluded in Section 5.

\section{Description and States of the System}

We consider a 2-out-of-3 system with three modes: normal, deterioration and failure. The deterioration mode consists of two consecutive stages: slow and fast. It is assumed that the system transits from normal to slow and later to fast deterioration with rate $\delta_{1}$ and $\delta_{2}$ respecttively. It is also assumed that the two consecutive units never fail simultaneously. Whenever the system deteriorate with rate $\delta_{1}$, minor minimal maintenance is invoke with rate $\mu_{1}$ to regain the system to its early stage prior to slow deterioration stage or the deterioration will be faster with rate $\delta_{2}$ where major minimal maintenance will be done with rate $\mu_{2}$. Unit I fail with rate $\beta_{1}$ and is under minimal repair with rate $\alpha_{1}$ and unit III is switch on. It is assumed that the switch from standby to operation is perfect. Similarly, unit II fails with rate $\beta_{2}$ and is minimally repaired with rate $\alpha_{2}$. The system failed when unit I and II have failed. The system is attended by one repair man.

\section{States of the System}

$S_{0}$ : Units I and II are in operation, unit III is in st Standby, the system is operational.

State $S_{1}$ : The system is under slow deterioration and is receiving minor minimal maintenance.

State $S_{2}$ : The system is under fast deterioration and is receiving major minimal maintenance.

$$
T=\left[\begin{array}{ccc}
-\left(\beta_{1}+\delta_{1}\right) & \mu_{1} & 0 \\
\delta_{1} & -\left(\beta_{1}+\mu_{1}+\delta_{2}\right) & \mu_{2} \\
0 & \delta_{2} & -\left(\beta_{1}+\mu_{2}\right) \\
\beta_{1} & \beta_{1} & 0 \\
0 & 0 & \beta_{1} \\
0 & 0 & 0
\end{array}\right.
$$

\subsection{System Availability Analysis}

For the availability case of Figure 1 using the initial condition in section 3 for this system,
State $S_{3}$ : Unit I failed and is under repair, units II and III are in operation, the system is in slow deterioration stage and Operational.

State $S_{4}$ : Unit I failed and is under repair, units II and III are in operation, the system is in fast deterioration stage and Operational.

State $S_{5}$ : Units II failed and is under repair, the system failed.

\section{Models Formulation}

Let $P(t)$ be the probability row vector at time $t$, then the initial conditions for this problem are as follows:

$$
\begin{aligned}
& P(0) \\
& =\left[P_{0}(0), P_{1}(0), P_{2}(0), P_{3}(0), P_{4}(0), P_{5}(0)\right] \\
& =[1,0,0,0,0,0]
\end{aligned}
$$

we obtain the following system of differential equations:

$$
\begin{aligned}
P_{0}^{\prime}(t)=- & \left(\beta_{1}+\delta_{1}\right) P_{0}(t)+\mu_{1} P_{1}(t)+\alpha_{1} P_{3}(t) \\
P_{1}^{\prime}(t)=- & \left(\beta_{1}+\mu_{1}+\delta_{2}\right) P_{1}(t) \\
& +\delta_{1} P_{0}(t)+\mu_{2} P_{2}(t)+\alpha_{1} P_{3}(t) \\
P_{2}^{\prime}(t)=- & \left(\beta_{1}+\mu_{2}\right) P_{2}(t)+\delta_{2} P_{1}(t)+\alpha_{1} P_{4}(t) \\
P_{3}^{\prime}(t)=- & \left(\beta_{2}+\delta_{2}+2 \alpha_{1}\right) P_{3}(t)+\beta_{1} P_{0}(t) \\
& +\beta_{1} P_{1}(t)+\mu_{2} P_{4}(t)+\alpha_{2} P_{5}(t) \\
P_{4}^{\prime}(t)= & -\left(\beta_{2}+\alpha_{1}+\mu_{2}\right) P_{4}(t) \\
& +\beta_{1} P_{2}(t)+\delta_{2} P_{3}(t)+\alpha_{2} P_{5}(t) \\
P_{5}^{\prime}(t)=- & 2 \alpha_{2} P_{5}(t)+\beta_{2} P_{3}(t)+\beta_{2} P_{4}(t)
\end{aligned}
$$

The differential equations in (1) above is transformed into matrix as

$$
P^{\prime}=T P
$$

where

$$
\left.\begin{array}{ccc}
\alpha_{1} & 0 & 0 \\
\alpha_{1} & 0 & 0 \\
0 & \alpha_{1} & 0 \\
-\left(\beta_{2}+\delta_{2}+2 \alpha_{1}\right) & \mu_{2} & \alpha_{2} \\
\delta_{2} & -\left(\beta_{2}+\alpha_{1}+\mu_{2}\right) & \alpha_{2} \\
\beta_{2} & \beta_{2} & -2 \alpha_{2}
\end{array}\right]
$$

$$
\begin{aligned}
& P(0)=\left[P_{1}(0), P_{2}(0), P_{3}(0), P_{4}(0), P_{5}(0), P_{6}(0)\right] \\
& =[1,0,0,0,0,0,0]
\end{aligned}
$$


The system of differential equations in (1) for the system above can be expressed in matrix form as:

$$
\left[\begin{array}{c}
P_{0}^{\prime} \\
P_{1}^{\prime} \\
P_{2}^{\prime} \\
P_{3}^{\prime} \\
P_{4}^{\prime} \\
P_{5}^{\prime}
\end{array}\right]=\left[\begin{array}{cccccc}
-\left(\beta_{1}+\delta_{1}\right) & \mu_{1} & 0 & \alpha_{1} & 0 & 0 \\
\delta_{1} & -\left(\beta_{1}+\mu_{1}+\delta_{2}\right) & \mu_{2} & \alpha_{1} & 0 & 0 \\
0 & \delta_{2} & -\left(\beta_{1}+\mu_{2}\right) & 0 & \alpha_{1} & 0 \\
\beta_{1} & \beta_{1} & 0 & -\left(\beta_{2}+\delta_{2}+2 \alpha_{1}\right) & \mu_{2} & \alpha_{2} \\
0 & 0 & \beta_{1} & \delta_{2} & -\left(\beta_{2}+\alpha_{1}+\mu_{2}\right) & \alpha_{2} \\
0 & 0 & 0 & \beta_{2} & \beta_{2} & -2 \alpha_{2}
\end{array}\right]\left[\begin{array}{c}
P_{0} \\
P_{1} \\
P_{2} \\
P_{3} \\
P_{4} \\
P_{5}
\end{array}\right]
$$

Let $V$ be the time to failure of the system. The become zero, thus (2) becomes steady-state availability is given by

$$
A_{V}=P_{0}(\infty)+P_{1}(\infty)+P_{2}(\infty)+P_{3}(\infty)+P_{4}(\infty)
$$

$$
T P(\infty)=0
$$

In steady state, the derivatives of state probabilities

which in matrix form is

$$
\left[\begin{array}{cccccc}
-\left(\beta_{1}+\delta_{1}\right) & \mu_{1} & 0 & \alpha_{1} & 0 & 0 \\
\delta_{1} & -\left(\beta_{1}+\mu_{1}+\delta_{2}\right) & \mu_{2} & \alpha_{1} & 0 & 0 \\
0 & \delta_{2} & -\left(\beta_{1}+\mu_{2}\right) & 0 & \alpha_{1} & 0 \\
\beta_{1} & \beta_{1} & 0 & -\left(\beta_{2}+\delta_{2}+2 \alpha_{1}\right) & \mu_{2} & \alpha_{2} \\
0 & 0 & \beta_{1} & \delta_{2} & -\left(\beta_{2}+\alpha_{1}+\mu_{2}\right) & \alpha_{2} \\
0 & 0 & 0 & \beta_{2} & \beta_{2} & -2 \alpha_{2}
\end{array}\right]\left[\begin{array}{l}
P_{0} \\
P_{1} \\
P_{2} \\
P_{3} \\
P_{4} \\
P_{5}
\end{array}\right]=\left[\begin{array}{l}
0 \\
0 \\
0 \\
0 \\
0 \\
0
\end{array}\right]
$$

using the normalizing condition

$$
\begin{aligned}
& P_{0}(\infty)+P_{1}(\infty)+P_{2}(\infty)+P_{3}(\infty)+P_{4}(\infty)+P_{5}(\infty)=1 \\
& {\left[\begin{array}{cccccc}
-\left(\beta_{1}+\delta_{1}\right) & \mu_{1} & 0 & \alpha_{1} & 0 & 0 \\
\delta_{1} & -\left(\beta_{1}+\mu_{1}+\delta_{2}\right) & \mu_{2} & \alpha_{1} & 0 & 0 \\
0 & \delta_{2} & -\left(\beta_{1}+\mu_{2}\right) & 0 & \alpha_{1} & 0 \\
\beta_{1} & \beta_{1} & 0 & -\left(\beta_{2}+\delta_{2}+2 \alpha_{1}\right) & \mu_{2} & \alpha_{2} \\
0 & 0 & \beta_{1} & \delta_{2} & -\left(\beta_{2}+\alpha_{1}+\mu_{2}\right) & \alpha_{2} \\
1 & 1 & 1 & 1 & 1 & 1
\end{array}\right]\left[\begin{array}{l}
P_{0} \\
P_{1} \\
P_{2} \\
P_{3} \\
P_{4} \\
P_{5}
\end{array}\right]=\left[\begin{array}{l}
0 \\
0 \\
0 \\
0 \\
0 \\
1
\end{array}\right]}
\end{aligned}
$$

Expression for $A_{V}$ thus is:

$$
A_{V}=N_{2} / D_{2}
$$

$$
\begin{aligned}
N_{2}= & \alpha_{1} \alpha_{2}\left(4 \alpha_{1} \mu_{1} \mu_{2}+4 \mu_{1} \mu_{2}^{2}+4 \beta_{1} \mu_{1} \mu_{2}+3 \beta_{2} \mu_{1} \mu_{2}+2 \mu_{1} \mu_{2} \delta_{2}+2 \beta_{1} \beta_{2} \mu_{1}+2 \alpha_{1} \beta_{1} \mu_{2}+2 \beta_{1} \mu_{2}^{2}+2 \beta_{1} \mu_{2} \delta_{2}+\beta_{1} \beta_{2} \mu_{2}\right. \\
& \left.+2 \beta_{1}^{2} \mu_{2}+\beta_{1} \beta_{2} \delta_{2}+\beta_{1}^{2} \beta_{2}\right)+\alpha_{1} \alpha_{2}\left(4 \alpha_{1} \mu_{2} \delta_{1}+2 \alpha_{1} \beta_{1} \mu_{2}+4 \mu_{2}^{2} \delta_{1}+2 \beta_{1} \mu_{2}^{2}+4 \beta_{1} \mu_{2} \delta_{1}+2 \beta_{1}^{2} \mu_{2}+2 \mu_{2} \delta_{1} \delta_{2}+2 \beta_{1} \beta_{2} \mu_{2}\right. \\
& \left.+3 \beta_{2} \mu_{2} \delta_{1}+2 \beta_{1} \mu_{2} \delta_{2}+2 \beta_{1} \beta_{2} \delta_{1}+\beta_{1}^{2} \beta_{2}\right)+\alpha_{1} \alpha_{2}\left(\beta_{1} \beta_{2} \mu_{1}+2 \beta_{1} \mu_{1} \delta_{2}+2 \alpha_{1} \beta_{1} \delta_{2}+4 \alpha_{1} \delta_{1} \delta_{2}+2 \beta_{1} \mu_{2} \delta_{2}+4 \mu_{2} \delta_{1} \delta_{2}+2 \beta_{1}^{2} \delta_{2}\right. \\
& \left.+2 \beta_{1} \beta_{2} \delta_{2}+2 \delta_{1} \delta_{2}^{2}+2 \beta_{1} \delta_{2}^{2}+3 \beta_{2} \delta_{1} \delta_{2}+2 \beta_{1} \delta_{1} \delta_{2}+\beta_{1}^{2} \beta_{2}+\beta_{1} \beta_{2} \delta_{1}\right)+\alpha_{2} \beta_{1}\left(2 \alpha_{1} \mu_{1} \mu_{2}+2 \mu_{1} \mu_{2}^{2}+\beta_{2} \mu_{1} \mu_{2}+2 \beta_{1} \mu_{1} \mu_{2}\right. \\
& +\beta_{1} \beta_{2} \mu_{1}+2 \alpha_{1} \mu_{2} \delta_{1}+2 \alpha_{1} \beta_{1} \mu_{2}+2 \mu_{2}^{2} \delta_{1}+2 \beta_{1} \mu_{2}^{2}+2 \beta_{1} \mu_{2} \delta_{1}+2 \beta_{1}^{2} \mu_{2}+2 \beta_{1} \mu_{2} \delta_{2}+2 \mu_{2} \delta_{1}+\beta_{2} \mu_{2} \delta_{1}+\beta_{1} \beta_{2} \mu_{2}+\beta_{1} \beta_{2} \delta_{1} \\
& \left.+\beta_{1} \beta_{2} \delta_{2}+\beta_{2} \delta_{1} \delta_{2}+\beta_{1}^{2} \beta_{2}\right)+\alpha_{2} \beta_{1}\left(\beta_{2} \mu_{1} \mu_{2}+2 \mu_{1} \mu_{2} \delta_{2}+2 \beta_{1} \mu_{1} \delta_{2}+\beta_{1} \beta_{2} \mu_{1}+2 \alpha_{1} \beta_{1} \delta_{2}+4 \alpha_{1} \delta_{1} \delta_{2}+2 \beta_{1} \mu_{2} \delta_{2}+\beta_{2} \mu_{2} \delta_{1}\right. \\
& \left.+2 \mu_{2} \delta_{1} \delta_{2}+\beta_{1} \beta_{2} \mu_{2}+2 \beta_{1}^{2} \delta_{2}+\beta_{2} \delta_{1} \delta_{2}+2 \beta_{1} \delta_{1} \delta_{2}+\beta_{1} \beta_{2} \delta_{2}+2 \beta_{1} \delta_{2}^{2}+\beta_{1}^{2} \beta_{2}+2 \delta_{1} \delta_{2}^{2}+\beta_{1} \beta_{2} \delta_{1}\right)
\end{aligned}
$$




$$
\begin{aligned}
& D_{2}=2 \beta_{1}^{2} \beta_{2} \mu_{2} \delta_{2}+\alpha_{1} \beta_{1}^{2} \beta_{2} \delta_{2}+2 \alpha_{2} \beta_{1}^{2} \beta_{2} \delta_{2}+2 \alpha_{1} \alpha_{2} \beta_{1} \delta_{2}^{2}+4 \alpha_{1} \alpha_{2} \beta_{1}^{2} \delta_{2}+4 \alpha_{2} \beta_{1}^{2} \mu_{2} \delta_{2}+\beta_{1}^{2} \beta_{2} \delta_{2}^{2}+\beta_{1}^{2} \beta_{2}^{2} \delta_{2}+\beta_{1}^{3} \beta_{2} \delta_{2} \\
& +2 \alpha_{2} \beta_{1}^{2} \delta_{2}^{2}+2 \alpha_{2} \beta_{1}^{3} \delta_{2}+6 \alpha_{1} \alpha_{2} \beta_{1} \mu_{2} \delta_{2}+2 \alpha_{1} \alpha_{2} \mu_{1} \mu_{2} \delta_{2}+\beta_{1} \beta_{2} \delta_{1} \delta_{2}^{2}+\beta_{1} \beta_{2} \mu_{1} \mu_{2} \delta_{2}+2 \beta_{1} \beta_{2} \mu_{2} \delta_{1} \delta_{2}+2 \alpha_{2} \beta_{1}^{2} \mu_{1} \delta_{2} \\
& +2 \alpha_{2} \beta_{1} \mu_{1} \mu_{2} \delta_{2}+2 \alpha_{2} \beta_{1}^{2} \delta_{1} \delta_{2}+2 \alpha_{2} \beta_{1} \delta_{1} \delta_{2}^{2}+2 \alpha_{2} \beta_{1} \beta_{2} \delta_{1} \delta_{2}+4 \alpha_{2} \beta_{1} \mu_{2} \delta_{1} \delta_{2}+\beta_{1} \beta_{2}^{2} \delta_{1} \delta_{2}+\beta_{1}^{2} \beta_{2} \mu_{1} \mu_{2}+\beta_{1}^{2} \beta_{2} \mu_{2} \delta_{1} \\
& +2 \alpha_{2} \beta_{1}^{2} \mu_{2} \delta_{1}+2 \alpha_{2} \beta_{1}^{2} \beta_{2} \delta_{1}+2 \alpha_{2} \beta_{1}^{2} \beta_{2} \mu_{1}+2 \alpha_{2} \beta_{1}^{2} \mu_{1} \mu_{2}+2 \alpha_{2} \beta_{1}^{2} \beta_{2} \mu_{2}+2 \alpha_{2} \beta_{1} \mu_{1} \mu_{2}^{2}+\beta_{1} \beta_{2}^{2} \mu_{2} \delta_{1}+2 \alpha_{2} \beta_{1} \mu_{2}^{2} \delta_{1} \\
& +\beta_{1} \beta_{2} \mu_{1} \mu_{2}^{2}+\beta_{1} \beta_{2} \mu_{2}^{2} \delta_{1}+\beta_{1} \beta_{2}^{2} \mu_{1} \mu_{2}+\beta_{1}^{3} \beta_{2}^{2}+\beta_{1}^{3} \beta_{2} \mu_{2}+2 \alpha_{2} \beta_{1}^{3} \beta_{2}+2 \alpha_{2} \beta_{1}^{3} \mu_{2}+2 \alpha_{2} \beta_{1}^{2} \mu_{2}^{2}+\beta_{1}^{2} \beta_{2}^{2} \mu_{2}+\beta_{1}^{2} \beta_{2} \mu_{2}^{2} \\
& +\beta_{1}^{2} \beta_{2}^{2} \delta_{1}+\beta_{1}^{2} \beta_{2}^{2} \mu_{1}+2 \alpha_{2} \beta_{1} \beta_{2} \mu_{2} \delta_{1}+2 \alpha_{2} \beta_{1} \beta_{2} \mu_{1} \mu_{2}+\alpha_{1} \beta_{1}^{2} \beta_{2} \mu_{2}+6 \alpha_{1} \alpha_{2} \beta_{1}^{2} \mu_{2}+3 \alpha_{1} \alpha_{2} \beta_{1}^{2} \beta_{2}+4 \alpha_{1} \alpha_{2} \mu_{1} \mu_{2}^{2} \\
& +4 \alpha_{1} \alpha_{2} \beta_{1} \mu_{2}^{2}+4 \alpha_{1} \alpha_{2} \mu_{2}^{2} \delta_{1}+2 \alpha_{1}^{2} \alpha_{2} \beta_{1} \delta_{2}+4 \alpha_{1}^{2} \alpha_{2} \beta_{1} \mu_{2}+4 \alpha_{1}^{2} \alpha_{2} \delta_{1} \delta_{2}+4 \alpha_{1}^{2} \alpha_{2} \mu_{1} \mu_{2}+4 \alpha_{1}^{2} \alpha_{2} \mu_{2} \delta_{1}+2 \alpha_{1} \beta_{1} \beta_{2} \delta_{1} \delta_{2} \\
& +2 \alpha_{1} \alpha_{2} \beta_{1} \mu_{1} \delta_{2}+6 \alpha_{1} \alpha_{2} \beta_{1} \delta_{1} \delta_{2}+\alpha_{1} \beta_{1} \beta_{2} \mu_{1} \mu_{2}+\alpha_{1} \beta_{1} \beta_{2} \mu_{2} \delta_{1}+3 \alpha_{1} \alpha_{2} \beta_{2} \delta_{1} \delta_{2}+3 \alpha_{1} \alpha_{2} \beta_{2} \mu_{2} \delta_{1}+\alpha_{1} \alpha_{2} \beta_{2} \mu_{1} \mu_{2} \\
& +3 \alpha_{1} \alpha_{2} \beta_{1} \beta_{2} \delta_{2}+3 \alpha_{1} \alpha_{2} \beta_{1} \beta_{2} \mu_{1}+6 \alpha_{1} \alpha_{2} \beta_{1} \mu_{1} \mu_{2}+3 \alpha_{1} \alpha_{2} \beta_{1} \beta_{2} \delta_{1}+3 \alpha_{1} \alpha_{2} \beta_{1} \beta_{2} \mu_{2}+6 \alpha_{1} \alpha_{2} \beta_{1} \mu_{2} \delta_{1}
\end{aligned}
$$

\subsection{Busy Period Analysis}

Using the same initial condition in section 3 above as for the reliability case

$$
\begin{aligned}
& P(0)=\left[P_{1}(0), P_{2}(0), P_{3}(0), P_{4}(0), P_{5}(0), P_{6}(0)\right] \\
& =[1,0,0,0,0,0,0]
\end{aligned}
$$

$$
\left[\begin{array}{c}
P_{0}^{\prime} \\
P_{1}^{\prime} \\
P_{2}^{\prime} \\
P_{3}^{\prime} \\
P_{4}^{\prime} \\
P_{5}^{\prime}
\end{array}\right]=\left[\begin{array}{ccc}
-\left(\beta_{1}+\delta_{1}\right) & \mu_{1} & 0 \\
\delta_{1} & -\left(\beta_{1}+\mu_{1}+\delta_{2}\right) & \mu_{2} \\
0 & \delta_{2} & -\left(\beta_{1}+\mu_{2}\right) \\
\beta_{1} & \beta_{1} & 0 \\
0 & 0 & \beta_{1} \\
0 & 0 & 0
\end{array}\right.
$$

Let $V$ be the time to failure of the system. The steady-state busy period is given by

$$
B_{V}=P_{1}(\infty)+P_{2}(\infty)+P_{3}(\infty)+P_{4}(\infty)+P_{5}(\infty)
$$

In steady state, the derivatives of state probabilities and (4) and (5) the busy period is obtained as follows:

In the steady state, the derivatives of the state probabilities become zero and this will enable us to compute steady state busy period:

The system of differential equations in (1) for the system above can be expressed in matrix form as:

$$
\left[\begin{array}{cccccc}
-\left(\beta_{1}+\delta_{1}\right) & \mu_{1} & 0 & \alpha_{1} & 0 & 0 \\
\delta_{1} & -\left(\beta_{1}+\mu_{1}+\delta_{2}\right) & \mu_{2} & \alpha_{1} & 0 & 0 \\
0 & \delta_{2} & -\left(\beta_{1}+\mu_{2}\right) & 0 & \alpha_{1} & 0 \\
\beta_{1} & \beta_{1} & 0 & -\left(\beta_{2}+\delta_{2}+2 \alpha_{1}\right) & \mu_{2} & \alpha_{2} \\
0 & 0 & \beta_{1} & \delta_{2} & -\left(\beta_{2}+\alpha_{1}+\mu_{2}\right) & \alpha_{2} \\
0 & 0 & 0 & \beta_{2} & \beta_{2} & -2 \alpha_{2}
\end{array}\right]\left[\begin{array}{l}
P_{0} \\
P_{1} \\
P_{2} \\
P_{3} \\
P_{4} \\
P_{5}
\end{array}\right]=\left[\begin{array}{l}
0 \\
0 \\
0 \\
0 \\
0 \\
0
\end{array}\right]
$$

using the normalizing condition

$$
P_{0}(\infty)+P_{1}(\infty)+P_{2}(\infty)+P_{3}(\infty)+P_{4}(\infty)+P_{5}(\infty)=1
$$

$\left.\begin{array}{ccc}\alpha_{1} & 0 & 0 \\ \alpha_{1} & 0 & 0 \\ 0 & \alpha_{1} & 0 \\ -\left(\beta_{2}+\delta_{2}+2 \alpha_{1}\right) & \mu_{2} & \alpha_{2} \\ \delta_{2} & -\left(\beta_{2}+\alpha_{1}+\mu_{2}\right) & \alpha_{2} \\ \beta_{2} & \beta_{2} & -2 \alpha_{2}\end{array}\right]\left[\begin{array}{c}P_{0} \\ P_{1} \\ P_{2} \\ P_{3} \\ P_{4} \\ P_{5}\end{array}\right]$

become zero, thus (2) becomes

$$
T P(\infty)=0
$$

which in matrix form is

$$
\left[\begin{array}{cccccc}
-\left(\beta_{1}+\delta_{1}\right) & \mu_{1} & 0 & \alpha_{1} & 0 & 0 \\
\delta_{1} & -\left(\beta_{1}+\mu_{1}+\delta_{2}\right) & \mu_{2} & \alpha_{1} & 0 & 0 \\
0 & \delta_{2} & -\left(\beta_{1}+\mu_{2}\right) & 0 & \alpha_{1} & 0 \\
\beta_{1} & \beta_{1} & 0 & -\left(\beta_{2}+\delta_{2}+2 \alpha_{1}\right) & \mu_{2} & \alpha_{2} \\
0 & 0 & \beta_{1} & \delta_{2} & -\left(\beta_{2}+\alpha_{1}+\mu_{2}\right) & \alpha_{2} \\
1 & 1 & 1 & 1 & 1 & 1
\end{array}\right]\left[\begin{array}{l}
P_{0} \\
P_{1} \\
P_{2} \\
P_{3} \\
P_{4} \\
P_{5}
\end{array}\right]=\left[\begin{array}{l}
0 \\
0 \\
0 \\
0 \\
0 \\
1
\end{array}\right]
$$

we substitute (8) in the last row of (7) following [2,3,5]. The resulting matrix is 
In the steady state, the derivatives of the state probabilities become zero and this will enable us to compute

$$
\begin{aligned}
& B(\infty)=1-P_{0}(\infty) \\
& N_{3}=\alpha_{1} \alpha_{2}\left(4 \alpha_{1} \mu_{2} \delta_{1}+2 \alpha_{1} \beta_{1} \mu_{2}+4 \mu_{2}^{2} \delta_{1}+2 \beta_{1} \mu_{2}^{2}+4 \beta_{1} \mu_{2} \delta_{1}+2 \beta_{1}^{2} \mu_{2}+2 \mu_{2} \delta_{1} \delta_{2}+2 \beta_{1} \beta_{2} \mu_{2}\right. \\
& \left.+3 \beta_{2} \mu_{2} \delta_{1}+2 \beta_{1} \mu_{2} \delta_{2}+2 \beta_{1} \beta_{2} \delta_{1}+\beta_{1}^{2} \beta_{2}\right)+\alpha_{1} \alpha_{2}\left(\beta_{1} \beta_{2} \mu_{1}+2 \beta_{1} \mu_{1} \delta_{2}+2 \alpha_{1} \beta_{1} \delta_{2}+4 \alpha_{1} \delta_{1} \delta_{2}+2 \beta_{1} \mu_{2} \delta_{2}+4 \mu_{2} \delta_{1} \delta_{2}+2 \beta_{1}^{2} \delta_{2}\right. \\
& \left.+2 \beta_{1} \beta_{2} \delta_{2}+2 \delta_{1} \delta_{2}^{2}+2 \beta_{1} \delta_{2}^{2}+3 \beta_{2} \delta_{1} \delta_{2}+2 \beta_{1} \delta_{1} \delta_{2}+\beta_{1}^{2} \beta_{2}+\beta_{1} \beta_{2} \delta_{1}\right)+\alpha_{2} \beta_{1}\left(2 \alpha_{1} \mu_{1} \mu_{2}+2 \mu_{1} \mu_{2}^{2}+\beta_{2} \mu_{1} \mu_{2}+2 \beta_{1} \mu_{1} \mu_{2}+\beta_{1} \beta_{2} \mu_{1}\right. \\
& +2 \alpha_{1} \mu_{2} \delta_{1}+2 \alpha_{1} \beta_{1} \mu_{2}+2 \mu_{2}^{2} \delta_{1}+2 \beta_{1} \mu_{2}^{2}+2 \beta_{1} \mu_{2} \delta_{1}+2 \beta_{1}^{2} \mu_{2}+2 \beta_{1} \mu_{2} \delta_{2}+2 \mu_{2} \delta_{1} \delta_{2}+\beta_{2} \mu_{2} \delta_{1}+\beta_{1} \beta_{2} \mu_{2}+\beta_{1} \beta_{2} \delta_{1}+\beta_{1} \beta_{2} \delta_{2} \\
& \left.+\beta_{2} \delta_{1} \delta_{2}+\beta_{1}^{2} \beta_{2}\right)+\alpha_{2} \beta_{1}\left(\beta_{2} \mu_{1} \mu_{2}+2 \mu_{1} \mu_{2} \delta_{2}+2 \beta_{1} \mu_{1} \delta_{2}+\beta_{1} \beta_{2} \mu_{1}+2 \alpha_{1} \beta_{1} \delta_{2}+4 \alpha_{1} \delta_{1} \delta_{2}+2 \beta_{1} \mu_{2} \delta_{2}+\beta_{2} \mu_{2} \delta_{1}+2 \mu_{2} \delta_{1} \delta_{2}\right) \\
& \left.+\beta_{1} \beta_{2} \mu_{2}+2 \beta_{1}^{2} \delta_{2}+\beta_{2} \delta_{1} \delta_{2}+2 \beta_{1} \delta_{1} \delta_{2}+\beta_{1} \beta_{2} \delta_{2}+2 \beta_{1} \delta_{2}^{2}+\beta_{1}^{2} \beta_{2}+2 \delta_{1} \delta_{2}^{2}+\beta_{1} \beta_{2} \delta_{1}\right)+\beta_{1} \beta_{2}\left(\alpha_{1} \beta_{1} \mu_{2}+\beta_{1} \beta_{2} \mu_{2}+\beta_{1} \beta_{2} \mu_{1}\right. \\
& +\beta_{1} \mu_{1} \mu_{2}+2 \beta_{1} \mu_{2} \delta_{2}+\beta_{1} \beta_{2} \delta_{2}+\beta_{2} \mu_{1} \mu_{2}+\beta_{1} \mu_{1} \delta_{2}+2 \mu_{2} \delta_{1} \delta_{2}+\beta_{1} \delta_{2}^{2}+\alpha_{1} \beta_{1} \delta_{2}+\beta_{1}^{2} \delta_{2}+\mu_{1} \mu_{2} \delta_{2}+\alpha_{1} \mu_{1} \mu_{2}+\beta_{2} \mu_{2} \delta_{1} \\
& \left.+\beta_{1} \mu_{2} \delta_{1}+\beta_{1} \beta_{2} \delta_{1}+\beta_{2} \delta_{1} \delta_{2}+\beta_{1} \delta_{1} \delta_{2}+\delta_{1} \delta_{2}^{2}+\beta_{1} \mu_{2}^{2}+\beta_{1}^{2} \mu_{2}+\beta_{1}^{2} \beta_{2}+\mu_{1} \mu_{2}^{2}+\mu_{2}^{2} \delta_{1}+2 \alpha_{1} \delta_{1} \delta_{2}+\alpha_{1} \mu_{2} \delta_{1}\right)
\end{aligned}
$$

\subsection{Profit Analysis}

The system/units are subjected to minor and major minimal maintenance and corrective maintenance at failure as can be observed in states 1, 2, 3, 4, and 5. From Figure 1 the repairman is busy performing corrective maintenance action to the units/system at failure in states 1, 2, 3, 4 and 5. According to [1-3], the expected profit per unit time incurred to the system in the steady-state is given by:

Profit $=$ total revenue generated - accumulated cost incurred due maintenance/repairing the failed units.

$$
P F=C_{0} A(\infty)-C_{1} B(\infty)
$$

where $\mathrm{PF}_{2}$ : is the profit incurred to the system.

$C_{0}$ : is the revenue per unit up time of the system.

$C_{1}$ : is the cost per unit time which the system is under repair.

\section{Results and Discussions}

In this section, we numerically obtained the results for mean time to system failure, system availability, busy

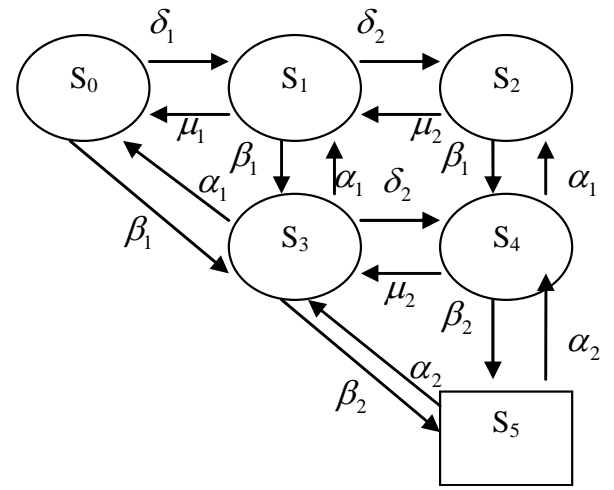

Figure 1. Transition diagram of the system. period and profit function for all the developed models. For the model analysis, the following set of parameters values are fixed throughout the simulations for consistency:

$$
\begin{gathered}
\beta_{1}=0.1, \beta_{2}=0.2, \alpha_{1}=0.4, \alpha_{2}=0.1, \delta_{1}=0.1, \\
\delta_{2}=0.1, \mu_{1}=0.3, \mu_{2}=0.4, C_{0}=50,000, C_{1}=10,000
\end{gathered}
$$

The impact of $\delta_{1}$ on profit can be observed in Figure 2. From this figure it is evident that the profit decreases as $\delta_{1}$ increases while in Figure 3, the increases with increase in $\mu_{1}$. Similar results can be observed in Figures 4 and 5 of profit with respect to $\delta_{2}$ and $\mu_{2}$. From these figures, the profit decreases as $\delta_{2}$ increases and increases with increase in $\mu_{2}$. Results of profit with respect to $\beta_{1}$ is given in Figure 6. It is evident from Figure 6 that as $\beta_{1}$ increases, the profit decreases while from Figure 7, the profit increases with increase in $\alpha_{1}$.

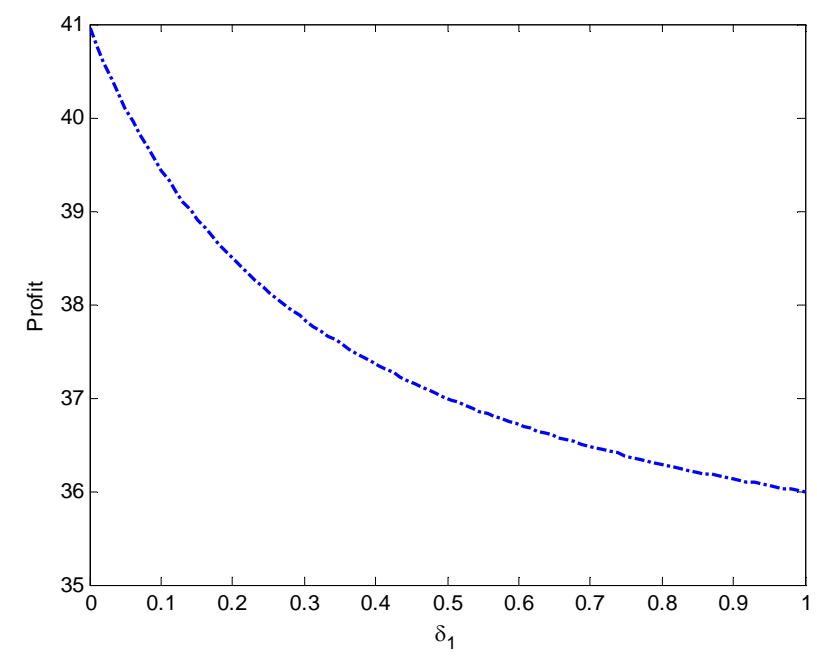

Figure 2. Effect of $\delta_{1}$ on Profit. 


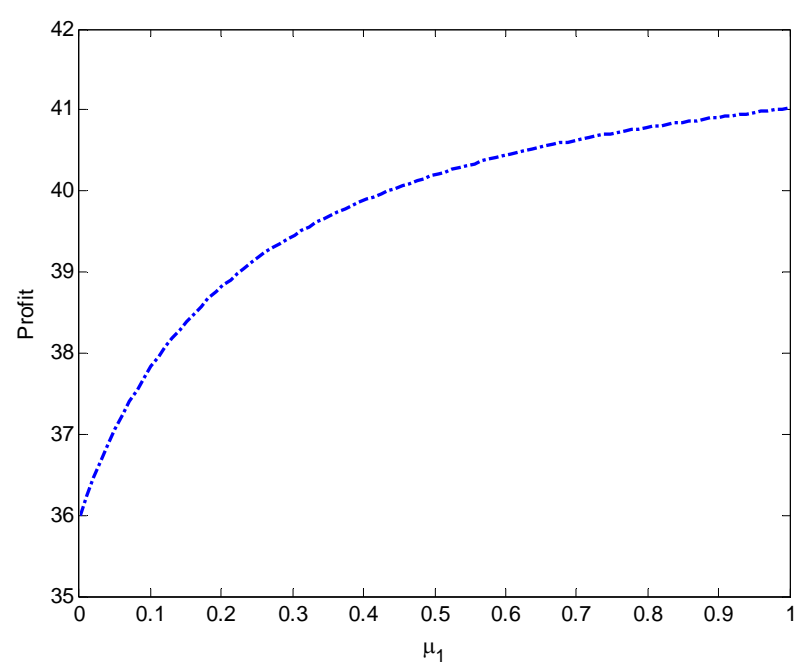

Figure 3. Effect of $\mu_{1}$ on Profit.

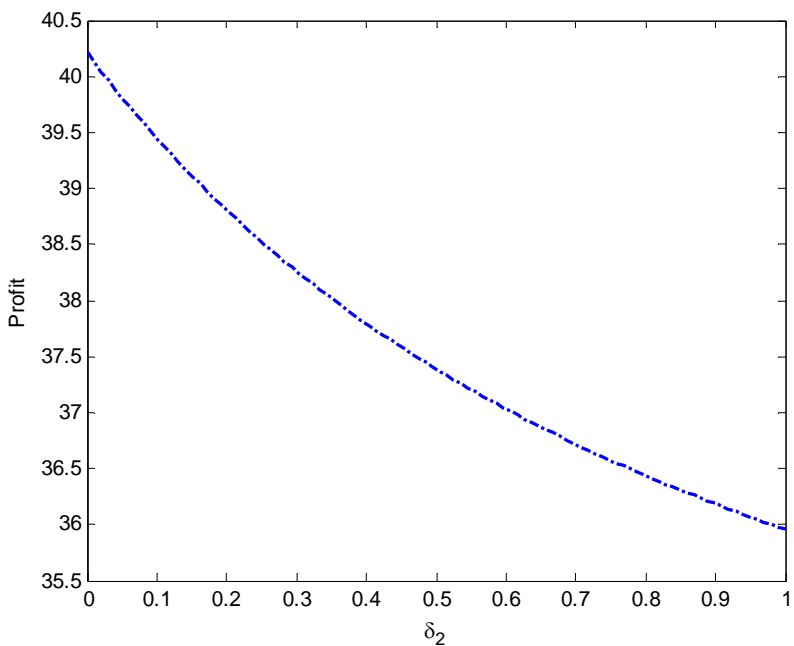

Figure 4. Effect of $\delta_{2}$ on Profit.

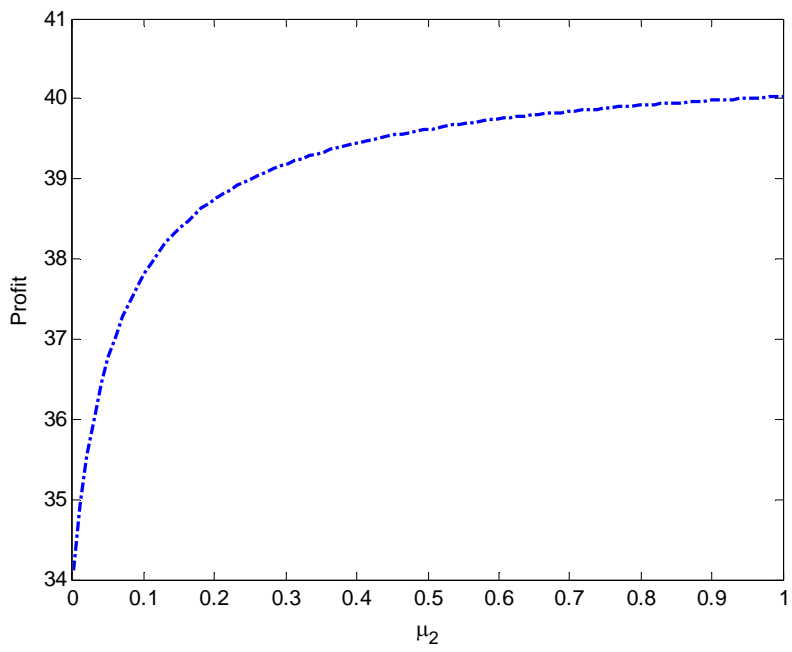

Figure 5. Effect of $\mu_{2}$ on Profit.

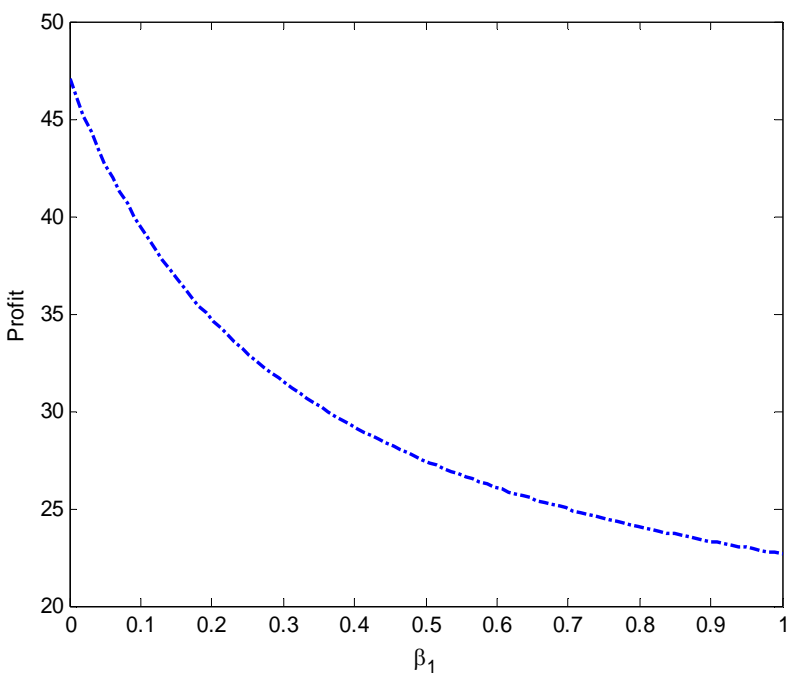

Figure 6. Effect of $\beta_{1}$ on Profit.

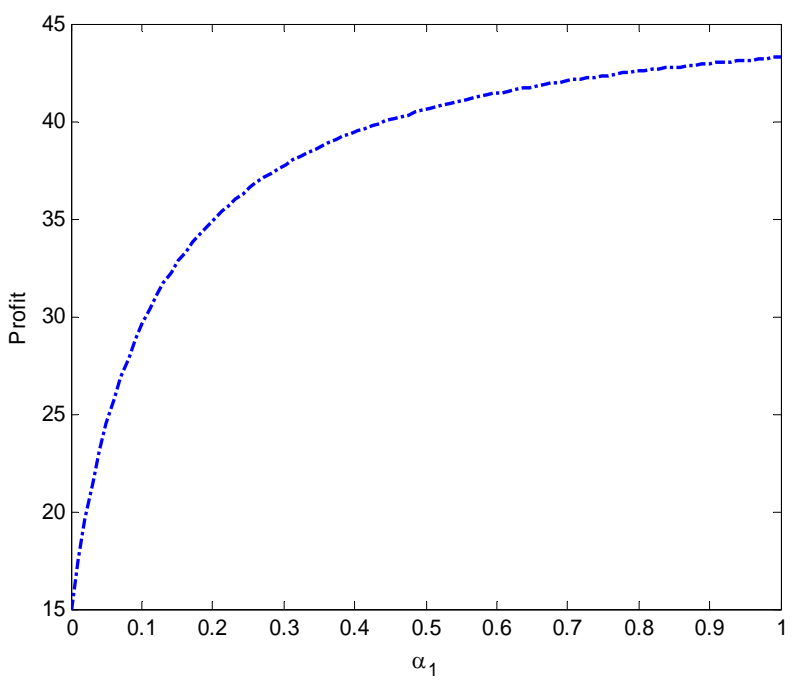

Figure 7. Effect of $\alpha_{1}$ on profit.

\section{Conclusion}

In this paper, we constructed a two-stage linear consecutive 2-out-of-3 system to study the impact of deterioration and maintenance on the generated profit. Explicit expressions of steady-state availability, busy period and profit function were derived. We performed numerical investigation to see the effect of slow deterioration, fast deterioration, minor minimal maintenance, major minimal maintenance, failure and repair rates on the generated profit. It is evident from the results obtained that repair rate, minor minimal maintenance rate and major minimal maintenance rates increase the profit generated while slow deterioration, fast deterioration and failure rate decrease the profit. It is evident from the results obtained that deterioration makes a tremendous effect on the generated revenue (profit). 


\section{Acknowledgements}

The authors are grateful to the anonymous reviewers for their constructive comments which have helped to improve the manuscript.

\section{REFERENCES}

[1] R. K. Bhardwaj and S. Chander, "Reliability and Cost Benefit Analysis of 2-out-of-3 Redundant System with General Distribution of Repair and Waiting Time,” DIAS Technology Review: The International Journal for Business \& IT, Vol. 4, No. 1, 2007, pp. 28-35.

[2] S. Chander and R. K. Bhardwai, "Reliability and Economic Analysis of 2-out-of-3 Redundant System with Priority to Repair," African Journal of Mathematics and Computer Science Research, Vol. 2, No. 11, 2009, pp. 230-236.

[3] R. K. Bhardwai and S. C. Malik, "MTSF and Cost Effectiveness of 2-out-of-3 Cold Standby System with Probability of Repair and Inspection,” International Journal of Engineering Science and Technology, Vol. 2, No. 1, 2010, pp. 5882-5889.

[4] K. Wang, C. Hsieh and C. Liou, "Cost Benefit Analysis of Series Systems with Cold Standby Components and a Repairable Service Station,” Journal of Quality Technology and Quantitative Management, Vol. 3, No. 1, 2006, pp. 77-92.
[5] K. M. El-Said, "Cost Analysis of a System with Preventive Maintenance by Using Kolmogorov’s forward Equations Method," American Journal of Applied Sciences, Vol. 5, No. 4, 2008 , pp. 405-410. doi:10.3844/ajassp.2008.405.410

[6] M. Y. Haggag, "Cost Analysis of a System Involving Common Cause Failures and Preventive Maintenance," Journal of Mathematics and Statistics, Vol. 5, No. 4, 2009, pp. 305-310. doi:10.3844/jmssp.2009.305.310

[7] K. H. Wang and C. C. Kuo, "Cost and Probabilistic Analysis of Series Systems with Mixed Standby Components," Applied Mathematical Modelling, Vol. 24, 2000, pp. 957-967. doi:10.1016/S0307-904X(00)00028-7

[8] K. C. Wang, Y. C, Liou and W. L. Pearn, "Cost Benefit Analysis of Series Systems with Warm Standby Components and General Repair Time,” Mathematical Methods of Operation Research, Vol. 61, 2005, pp. 329-343. doi:10.1007/s001860400385

[9] M. A. Hajeeh, "Availability of a System with Different Repair Options,” International Journal of Mathematics in Operational Research, Vol. 4, No. 1, 2012, pp. 41-55. doi:10.1504/IJMOR.2012.044472

[10] H. S. Fathabadi and M. Khodaei, "Reliability Evaluation of Network Flows with Stochastic Capacity and Cost Constraint," International Journal of Mathematics in Operational Research, Vol. 4, No. 4, 2012, pp. 439-452. doi:10.1504/IJMOR.2012.048904 